\section{ELEVATED CYSTEINE-RICH PROTEIN 61 IN SYSTEMIC LUPUS ERYTHEMATOSUS-ASSOCIATED PULMONARY ARTERIAL HYPERTENSION}

${ }^{1} Y$ Fan*, ${ }^{2} \mathrm{~J}$ Qian, ${ }^{1} \mathrm{Y}$ Hao, ${ }^{2} \mathrm{M}$ Li, ${ }^{3} \mathrm{~L}$ Gao, ${ }^{2} \mathrm{X}$ Zeng, ${ }^{1} \mathrm{Z}$ Zhang. ${ }^{1}$ Peking University First Hospital, Department of Rheumatology and Clinical Immunology, Beijing, China; ${ }^{2}$ Peking Union Medical College Hospital- Peking Union Medical College and Chinese Academy of Medical Sciences- Key Laboratory of Rheumatology and Clinical Immunology- Ministry of Education, Department of Rheumatology, Beijing, China; ${ }^{3}$ Beijing Shijitan Hospital- Capital Medical University- Peking University Ninth Hospital, Department of Rheumatology and Immunology, Beijing, China

\subsection{6/lupus-2017-000215.156}

Background and aims Previous study has demonstrated Cysteine-rich protein 61(Cyr61) was highly expressed in systemic lupus erythematosus(SLE) patients.However,the role of Cyr61 in pulmonary arterial hypertension (PAH) remains unknown. This study aimed to explore the value of Cyr61 for PAH in SLE patients by comparing the plasma Cyr61 levels in SLE patients with/without $\mathrm{PAH}$.

Methods Plasma samples from two tertiary medical centerswereobtained from 54 patients with definite SLE-PAH, 52 age, gender and SLEDAI matched SLE patients without PAH, and 54 age and gender matched healthy controls. Plasma Cyr61 concentration was measured by enzyme-linked immunosorbent assay.

Results Plasma Cyr61 concentration in SLE-PAH patients was significantly higher than the matched SLE patients and healthy controls(median(IQR): $\quad 172.5 \quad(143.8, \quad 218.2), \quad 124.9$ (104.1,154.7), 58.17 (28.9, 80.4) respectively, $\mathrm{P}<0.001)$ (Figure 1). The sensitivity and specificity of Cyr61 in predicting the presence of PAH in entire SLE patients were $79.6 \%$ and $67.3 \%$. Receiver operating characteristic curve analysis showed the area under the curve was 0.757 (95\% CI:0.662 0.852), with $140.6 \mathrm{pg} / \mathrm{ml}$ as the cutoff concentration (Figure 2). Further multivariate logistic regression analysesrevealed high Cyr61 level $(>140.6)$ is an independent risk factor for SLE patients to develop PAH (OR:7.822 (95\% CI:2.224 41.138)) (Table 1). Additionally, weak to moderate positive correlations were observed between Cyr61 concentration and serositis,

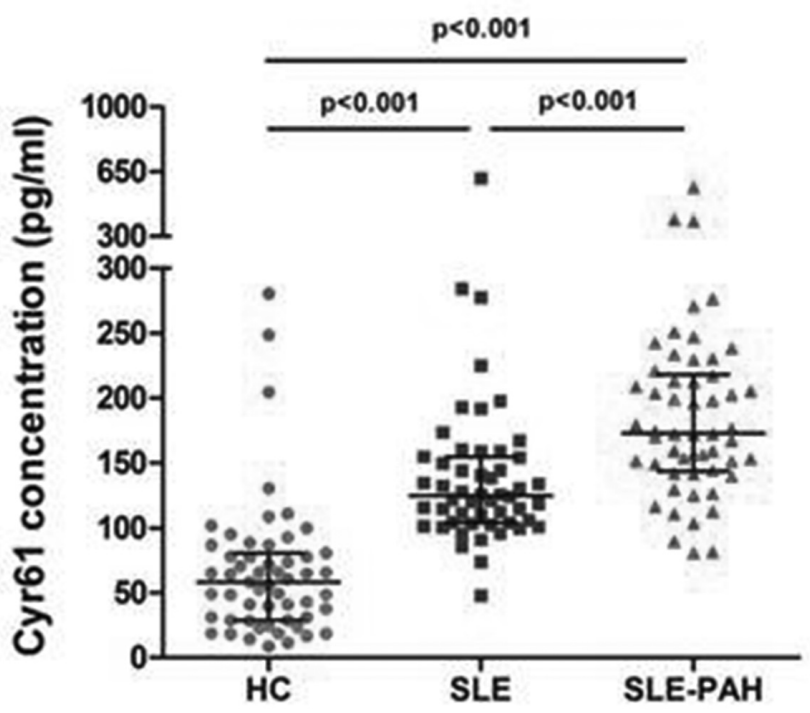

Abstract 156 Figure 1

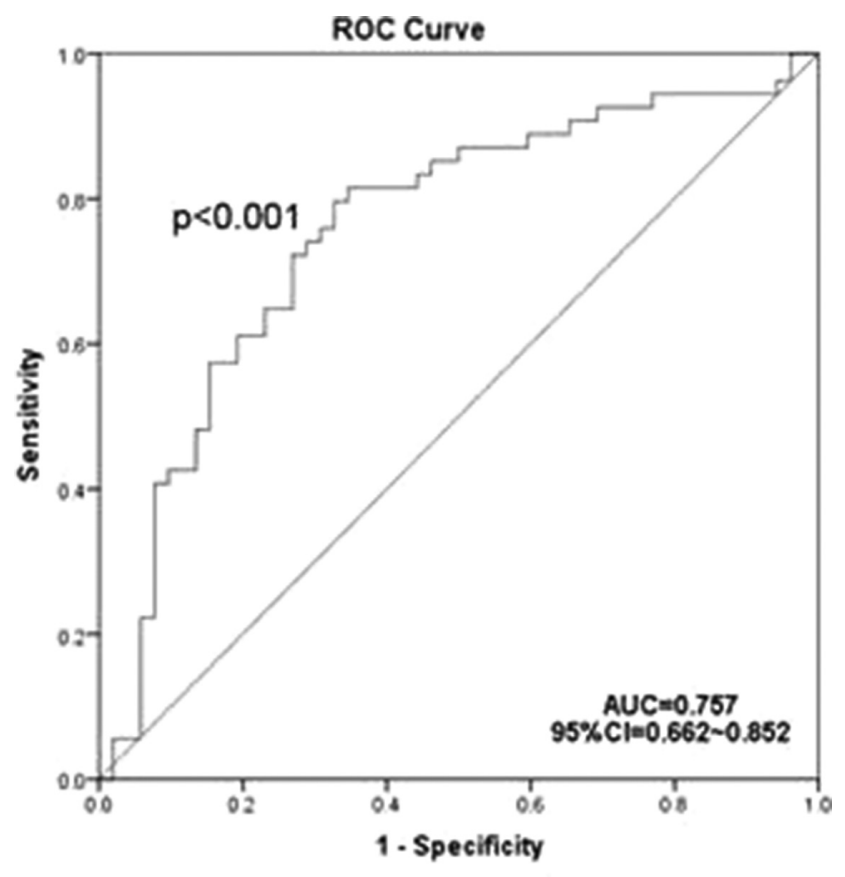

Abstract 156 Figure 2

haematological involvement, red blood cell distribution width, right ventricular systolic pressure and right ventricular diameter measured by echocardiography in entire SLE population. Conclusions Plasma Cyr61 level was significantly higher in SLE-PAH patients than SLE patients without PAH. Cyr61 may be used as a biomarker for PAH complication in SLE patients.

\section{ANTIBODIES TOWARDS ATP-BINDING CASSETTE TRANSPORTER ABCA1: A NEW MECHANISM FOR ATHEROSCLEROSIS IN SLE?}

1,2M Fernandes das Neves*, ${ }^{2} \mathrm{JR}$ Batuca, ${ }^{1} \mathrm{~F}$ Batista, ${ }^{1} \mathrm{C}$ Favas, ${ }^{1} \mathrm{C}$ Henriques, $1,2 \mathrm{~J}$ Delgado Alves. ${ }^{1}$ Hospital Prof. Doutor Fernando da Fonseca, UDIMS/Medicina IV, Amadora, Portugal; ${ }^{2}$ NOVA Medical School/Faculdade de Ciências Médicas- Universidade Nova de Lisboa, CEDOC, Lisboa, Portugal

\subsection{6/lupus-2017-000215.157}

Background and aims Systemic Lupus Erythematosus (SLE) is considered an independent risk factor for cardiovascular disease. ATP-binding cassette transporter ABCA1, also known as the cholesterol efflux regulator protein, is a ubiquitous cholesterol transporter that is highly expressed in macrophages. Its main function is to donate cholesterol to apolipoproteinA-I in lipid-poor HDL particles. As such, ABCA1 closely influences HDL levels and its role in atherosclerosis has been increasingly studied.

This study was undertaken to determine if antibodies against ABCA1 can be detected in patients with SLE.

Methods Serum from 48 patients were compared with an age and sex-matched control group. Patients were divided in groups A (13 patients) and B (35 patients), respectively with up to 3 and at least 4 SLICC classification criteria (2012). IgG anti-ABCA1 and anti-HDL antibodies were assessed by home-made ELISAs. Plasma lipid profile was determined by standard enzymatic techniques. 
Abstract 156 Table 1 Multivariate logistic regression analysis

\begin{tabular}{lllll}
\hline \multicolumn{1}{c}{ Variables } & OR & \multicolumn{2}{c}{$95 \%$ CI } & Pvalue \\
\hline High Cyr61 level & 7.822 & 2.224 & 41.138 & 0.002 \\
Serositis & 8.244 & 1.854 & 36.663 & 0.006 \\
Anti-RNPpositivity & 3.902 & 1.067 & 14.267 & 0.040 \\
Renal disorders & 0.174 & 0.044 & 0.689 & 0.013 \\
Anti-dsDNA positivity & 0.171 & 0.046 & 0.636 & 0.008 \\
\hline
\end{tabular}

Results Group A did not differ from control group. Group B had higher titers of anti-ABCA1 antibodies $(p=0.004)$, with 4 patients showing positive anti-ABCA1 titers (11.4\%). In group $\mathrm{B}$, anti-ABCA1 antibodies titers tend to negatively correlate with HDL $(p=0.06)$ and apoliporoteinA-I levels $(p=0.04)$. In both groups, anti-HDL antibodies did not correlate with antiABCA1 titers.

Conclusions This is the first report of naturally occurring antibodies against ABCA1. These antibodies are increased in patients with SLE with at least 4 SLICC classification criteria. Future studies will determine their pathogenic role.

This work was supported by the Portuguese Foundation for Science and Technology via the post-doctoral SFRH/BPD/ 112411/2015 and Fundação Oriente.

\section{CONTRIBUTORS TO DISEASE FLARES AMONG FILIPINO PATIENTS WITH SLE}

ME Fernandez, MFJ Edar, S Navarra. University of Santo Tomas Hospital, Internal MedicineRheumatology, Manila, Philippines

\subsection{6/lupus-2017-000215.158}

Background and aims SLE disease flares significantly contribute to morbidity and mortality in SLE. This study describes the characteristics and contributing factors to disease flares in a cohort of Filipino SLE patients.

Methods Included were SLE patients with disease flare seen consecutively from January 2012 to December 2015 at the Lupus Clinics of University of Santo Tomas (UST) Hospital, Manila, Philippines. Demographics, organ involvement and SLE flare severity by SELENA-SLEDAI Flare Index (SFI) were described. Contributing factors to flare were identified based on physician assessment notes and patient responses to the Life Experiences Survey (LES).

Results There were 100 disease flares in 73 patients (68 females). Mean age at SLE diagnosis was 25.15 \pm 8.57 years (4-43), disease duration at each flare occurrence was 7.40 $\pm 4.42(<1-21)$ years. Flares were severe in 60 , mild to moderate in 40. Organ involvement included renal in 47 (32.41\%), mucocutaneous 40 (27.59\%), musculoskeletal 26 (17.93\%), vasculitis 11 (7.59\%), hematologic 11 (7.59\%), neurologic 6 (4.14\%), ocular $(32.07 \%)$ and cardiopulmonary 1
(0.68\%). Stress and infection in 35 (30.97\%) each, were the leading contributors to flare. Others included non-compliance in $18(15.93 \%)$, excess sun exposure 7 (6.20\%), pregnancy 3 (2.66\%), fetal loss $3(2.66 \%)$, surgery and drugs during 1 $(0.88 \%)$ flare occurrence each, and unknown in $10(8.85 \%)$.

Conclusions Renal, mucocutaneous and musculoskeletal systems were most commonly involved in a disease flare. Stress and infection were the leading factors contributing to a flare. These findings underscore need for holistic management approach in SLE, integrating effective disease control with patient education

\section{NEUTROPHIL-TO-LYMPHOCYTE RATIO AND SYSTEMIC LUPUS ERYTHEMATOSUS: A NEW PARAMETER FOR DISEASE ACTIVITY ASSESSMENT?}

${ }^{1} \mathrm{H}$ Gunawan*, ${ }^{1} \mathrm{~A}$ Awalia, ${ }^{1} \mathrm{~J}$ Soeroso. ${ }^{1}$ Airlangga University, Internal Medicine Rheumatology Division, Surabaya, Indonesia

\subsection{6/lupus-2017-000215.159}

Background and aims Systemic Lupus Erythematosus (SLE) is a complex autoimmune disease characterised by enhanced apoptosis in conjunction with high levels of autoantibodies and deregulated cytokine productions. It contributes to immune dysfunction, tissue inflammation, and organ damage. Neutrophil to Lymphocyte ratio (NLR) is a promising marker in SLE, but its relationships with SLE's disease activity in Indonesian population has not been evaluated.

To investigate the correlation between NLR and SLE disease activity.

Methods A cross sectional study with consecutive sampling technique was conducted with 70 Indonesian SLE patients in Dr. Soetomo Hospital Surabaya at May 2016-July 2016. Peripheral blood count was evaluated during assessment with flowcytometry technique and disease activity was evaluated with Systemic Lupus Activity Measure (SLAM) score. Data analysis was done with Spearman's correlation rank test and ROC curve using SPSS v22.0.

Results There were 19 patients with SLAM score $<7$ (group 1) and 51 patients with SLAM score $\geq 7$ (group 2). We found that lymphocyte count and NLR was statistically significant between groups $\left[\begin{array}{lllll}1.58 & (0.66-2.73)\end{array}\right)$ vs $0.95 \quad(0.14-3.49)$; 